\title{
Caracterización hidroquímica del complejo de humedales El Yali, Chile Central
}

\author{
$\mathrm{M}^{\mathrm{a}}$ Rosario Vidal-Abarca ${ }^{1, *}, \mathrm{M}^{\mathrm{a}}$ Luisa Suárez ${ }^{1}$, Ricardo Figueroa ${ }^{2}$, Mariela Enríquez ${ }^{2}$, Victoria \\ García $^{1}$, Carmen Dominguez ${ }^{1}, \mathrm{M}^{\mathrm{a}}$ Isabel Arce ${ }^{1}$ \\ 1 Departamento de Ecología e Hidrología. Universidad de Murcia. Campus de Espinardo. 30100 Murcia \\ (España). \\ ${ }^{2}$ Unidad de sistemas acuáticos, Centro EULA-Chile, Universidad de Concepción, Casilla 160 C; Concepción, \\ (Chile).
}

* Autor responsable de la correspondencia: charyvag@um.es

Recibido: $29 / 6 / 10$

Aceptado: $11 / 2 / 11$

\begin{abstract}
Hydrochemical characterization of El Yali wetlands complex: Central Chile

Within the wetlands of the semiarid region of Central Chile, The Yali wetlands complex is the most important because it is protected as a RAMSAR's wetland. However, information about their hydro-geomorphological characteristics is almost non existent. In this study, the hydrochemical characteristics of 20 sampling sites, surveyed in July 2008 and which include coastal lagoons, estuaries, streams, saltmarshes, and reservoirs, were analyzed. The hydrochemical complexity stands out in relation to the water salinity (range $=320-58800 \mu \mathrm{S} / \mathrm{cm}$ ) and ionic composition of the studied wetlands in relation to the relative importance of the dominant water flow that sustains these wetlands. In relation to trophic state, most of the studied wetland systems are eutrophic or hypertrophic (medium values for total nitrogen $=4.7 \mathrm{mg} / \mathrm{l}$; and soluble reactive phosphorus $=0.4 \mathrm{mg} / \mathrm{l}$ ) due to the existing use of land (eucalyptus forests, cattle farms and wastewater inputs).
\end{abstract}

Key words: Central Chile, Wetland "El Yali”, hydrochemical, salinity, hydrology.

\section{RESUMEN}

Caracterización hidroquímica del complejo de humedales El Yali, Chile Central

Dentro de los humedales de la región semiárida Central de Chile, destaca el complejo denominado El Yali, por su condición de protección como Humedal RAMSAR, del que apenas existe información sobre sus características hidrogeomorfologicas. En este estudio se analiza la hidroquímica de 20 puntos de muestreo, realizados en julio de 2008, incluyendo lagunas, estuarios, esteros, salinas y embalses. Destaca su complejidad hidroquímica en relación con la salinidad (rango $=320-58800 \mu \mathrm{S} / \mathrm{cm}$ ) y el contenido iónico de los humedales estudiados en función de la importancia relativa del flujo de agua dominante. En cuanto al estado trófico, la mayoría de los sistemas estudiados son eutróficos o hipertróficos (valores medios de nitrógeno total = $4.7 \mathrm{mg} / \mathrm{l}$; y SRP (fósforo reactivo soluble) = $0.4 \mathrm{mg} / \mathrm{l}$ ), como consecuencia del uso del territorio (plantaciones de eucaliptos, explotaciones ganaderas y vertidos orgánicos).

Palabras clave: Chile Central, humedal El Yali, hidroquímica, salinidad, hidrología.

\section{INTRODUCCIÓN}

Los humedales constituyen uno de los tipos de ecosistemas más diversos del planeta (Daniels \&
Cumming, 2008). Aunque a nivel mundial no alcanzan el $3 \%$ de su superficie, contribuyen en más del $40 \%$ a los servicios que proporcionan a la humanidad (Zedler \& Kercher, 2005). No 
obstante, su tratamiento por parte del hombre no ha sido equilibrado a juzgar por la cantidad de humedales que han desaparecido y por la variedad de impactos y agresiones a las que han sido sometidos (Millennium Ecosystem Assesssment, 2005, Zedler \& West, 2008). Según Mitsch (2005) más del $50 \%$ de los humedales del planeta se han perdido en la era moderna. Este hecho responde a que, en general, los humedales han sido considerados sistemas insalubres, fuente de infecciones y transmisores de enfermedades (Casado \& Montes, 1995) y por tanto, pobremente valorados como fuente de recursos tangibles (pesca, caza, sal, recreo, turismo, etc.) y potenciales (control de nutrientes, protección frente a inundaciones, mitigación del cambio global, etc.) (Russo, 2008).

Debido a la diversidad de climas y al accidentado modelado del terreno, Chile cuenta con una gran cantidad de humedales que, si no son especialmente destacables por su tamaño, sí lo son por su singularidad y variedad (Mardones, 1999, Mardones \& Reuther, 1999, Parra et al., 2003, Figueroa et al., 2009). De hecho, algunos de ellos han sido reconocidos a nivel internacional y hoy Chile cuenta con 9 sitios Ramsar (CONAMA, 2005). El problema es que la gran mayoría de ellos carecen de programas de gestión, en buena parte debido a la ausencia de estudios sobre su dinámica hidrológica, biológica y ecológica, lo que dificulta plantear estrategias conjuntas de explotación-conservación.

La región semiárida de Chile es de especial interés dado que la estructura del paisaje permite la formación de sistemas lacustres y lagunas costeras ligadas a la dinámica marina que configura complejos de humedales de enorme singularidad hidrológica e hidroquímica. En este territorio se localiza El Yali, que constituye el complejo de humedales más importante de Chile Central y declarado sitio Ramsar en 1996. El único trabajo referido exclusivamente a la laguna costera del complejo El Yali es el de Dussaillant et al., (2009), que analiza la dinámica hidroquímica a diferentes escalas temporales.

Un estudio reciente de Figueroa et al. (2009) sobre los humedales de Chile central, que incluía 8 lagunas del complejo El Yali, reveló su enorme complejidad hidroquímica, debido a los valores extremos de conductividad que presentaban sus aguas (entre 52200 y $1286 \mu \mathrm{S} / \mathrm{cm}$ ), y la importancia de conocer el origen de los flujos de agua para el entendimiento de su dinámica natural. En efecto, muchos autores hacen referencia a que la singularidad hidrológica determina los procesos químicos, biológicos y ecológicos, factores determinantes para entender el funcionamiento de los humedales (e.g. Cole et al., 1997; Mitsch \& Gosselink, 2000).

Así, el principal objetivo de este trabajo es establecer una aproximación a la hidroquímica superficial de los humedales del complejo El Yali. El estudio de distintas variables físico-químicas del del agua y su interpretación en función de diferentes variables geográficas ayudan a entender el origen y dominancia de los flujos superficiales y/o subterráneos sobre la dinámica y características hidroquímicas de los cuerpos de agua estudiados.

\section{METODOLOGÍA}

\section{Área de estudio}

El complejo de humedales El Yali se localiza en $\mathrm{V}$ región de Chile (Fig. 1) a $35 \mathrm{~km}$ al sur de Santo Domingo y a $135 \mathrm{~km}$ al sur de Valparaíso. Cubre una superficie de 11500 ha, de las que 520.37 ha están protegidas como Reserva Nacional desde 1996, que son gestionadas por el CONAF (Corporación Nacional Forestal). Este complejo de humedales incluye una amplia variedad de cuerpos de agua (lechos y riberas de ríos, lagunas, embalses, suelos pantanosos y vegas, salinas artificiales, desembocaduras de esteros y dunas), con un fuerte dinamismo estacional (verano-invierno), anual y plurianual debido a los ciclos del El NIÑO-Oscilación del Sur (EN$\mathrm{SO})$. Se caracteriza por un clima mediterráneo semiárido con influencia oceánica, con nubosidad matinal en la costa casi todo el año. La precipitación media anual es de $481 \mathrm{~mm}$, con valores extremos de 779.5 y $140.5 \mathrm{~mm}$, donde el $90 \%$ de las lluvias se concentran entre los meses de mayo y agosto (Fernández, 2004). La temperatura media anual es de $13.2^{\circ} \mathrm{C}$ y la evapotranspiración de unos $1000 \mathrm{~mm}$ anuales (DGA, 2005).

La hidrografía del complejo de humedales El Yali incluye territorios de 5 cuencas hidrográfi- 


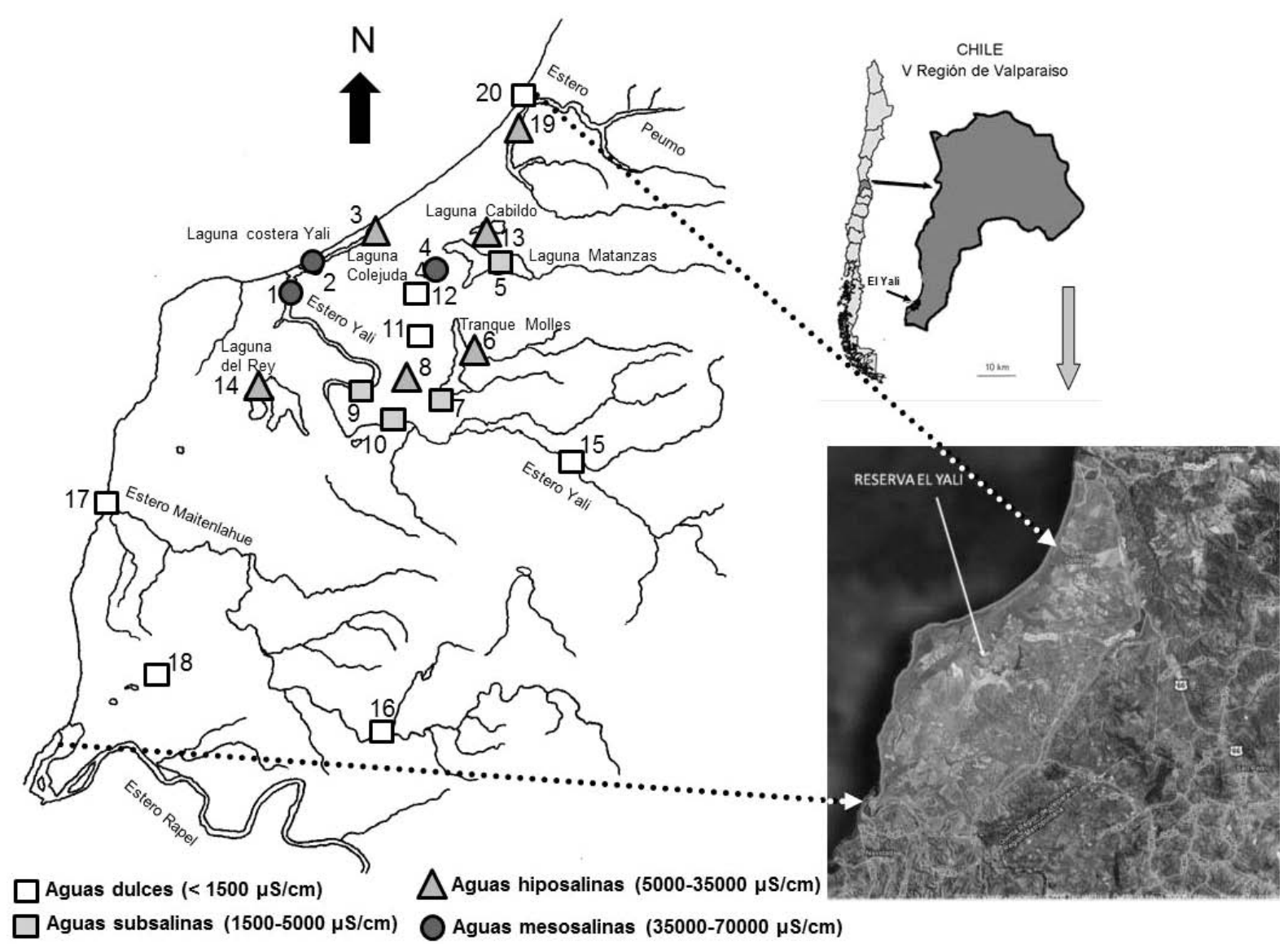

Figura 1. Localización geográfica y puntos de muestreo del complejo de humedales El Yali. Se señala el rango de los valores de conductividad. Geographical location and sampling sites of the Yali wetlands complex. The range of conductivity values is shown.

cas, la mayor y más importante es la del Estero El Yali. Al norte, la Cuenca de la Quebrada de Las Rosas o Las Monjas, prácticamente endorreica, alimenta la Laguna de Matanzas, una depresión natural de unos 10-15 m de profundidad y la Cuenca del Estero El Peumo, la más pequeña. Finalmente, al sur del Yali se encuentra la Cuenca del Estero Maitenlahue, que desemboca formando la laguna del Mostazal (Biblioredes, 2009) (Fig. 1).

En cuanto al origen del agua, el complejo El Yali incluye ecosistemas de origen marino, estuarino, lacustre y ribereño, además de salinas artificiales que ocasionalmente se inundan durante el otoño e invierno, pudiendo mantenerse activas hasta primavera para su explotación y varios embalses. Todo el complejo presenta una condición edáfica interna de mal drenaje, estando el nivel freático prácticamente en la superficie de terreno.
El complejo del Yali presenta una vegetación del tipo matorral espinoso del secano costero (Gajardo, 1994), único de su clase en América del Sur (Dinerstein et al., 1995). A orillas del mar predominan mayoritariamente especies suculentas sobre sustrato de arena, vegetación de pajonales, y pequeños remanentes de bosque nativo tipo esclerófilo. En las riberas de muchas de las lagunas hay plantaciones de eucalipto.

Únicamente la zona de reserva está destinada a la protección y conservación de la flora y fauna silvestre. El resto, sobre todo algunas lagunas, se utilizan como lugares de recreo y las salinas, para la extracción de sal. El área circundante a la reserva es de propiedad privada y presentan usos tradicionales principalmente agrícola y ganadero. Desde tiempos históricos, el Yali ha sufrido amenazas y agresiones por la acción 
antrópica: modificación del hábitat, extracción y desviación de aguas para uso agrícola, reducción de la vegetación nativa por incendios forestales, deforestación para uso agrícola y silvoagropecuario, construcción de inmuebles, acción de cazadores y pescadores furtivos o ilegales, movimiento de vehículos de doble tracción en la zona costera, vuelos rasantes de aeronaves civiles y militares, instalación de cableados eléctricos que causan colisiones con algunas especies de aves acuáticas, etc. Actualmente, hay que destacar la transformación de grandes superficies de terreno para la plantación de eucalipto y los impactos derivados de la entrada de residuos líquidos a algunas lagunas procedentes de una importante industria avícola desde 1991.

\section{Selección de las estaciones de muestreo y periodo de estudio}

Para el presente estudio se seleccionaron un total de 20 puntos de muestreo en lagunas, estuarios, esteros, salinas y embalses del Complejo El Yali. La dificultad de acceso a terrenos privados no permitió muestrear un número mayor de cuer- pos de agua. Para acceder a la Reserva, se obtuvo un permiso especial de CONAF. El periodo de muestreo abarcó del 22 al 27 de julio de 2008, correspondiente a la fase húmeda (otoño-invierno) del ciclo hidrológico anual. Todos los puntos de muestreo fueron georeferenciados y se recogió información geográfica sobre su tipología, hidrología, usos del suelo, unidades paisajísticas e impactos. De igual manera, se anotó el tipo de formación de la vegetación de ribera y acuática dominante en cada cuerpo de agua. En la Tabla 1 se presentan las características geográficas y tipología de los humedales estudiados y en la figura 1 , su localización geográfica.

\section{Variables físico-químicas}

En todos los humedales muestreados se recogió información de distintas variables del medio físico: origen del agua (aproximación según valores de salinidad), profundidad y anchura de la lámina de agua, el tipo de sustrato dominante (cualitativo). In situ se midió temperatura del aire y del agua, $\mathrm{pH}$ (pH-metro Schott Mod. Handylab CG$836 / 2046)$, conductividad eléctrica ( $\mu \mathrm{S} / \mathrm{cm}$, con-

Tabla 1. Características geográficas, físicas, hidrológicas y origen del agua de los humedales prospectados en el complejo El Yali. Geographical, physical, hydrological characteristics and origin of the water of the studied wetlands in the El Yali weland's complex.

\begin{tabular}{|c|c|c|c|c|c|c|c|c|c|c|c|c|}
\hline \multirow[b]{2}{*}{$\mathbf{N}^{\mathbf{o}}$} & \multirow[b]{2}{*}{ Nombre } & \multirow[b]{2}{*}{ Fecha } & \multicolumn{2}{|c|}{ Coordenadas } & \multirow{2}{*}{$\begin{array}{c}\text { Altitud } \\
\text { (m) }\end{array}$} & \multirow{2}{*}{$\begin{array}{c}\text { Distancia } \\
\text { al mar (m) }\end{array}$} & \multirow{2}{*}{$\begin{array}{c}\text { Ancho } \\
\text { (m) }\end{array}$} & \multirow{2}{*}{$\begin{array}{l}\text { Longitud } \\
\text { (m) }\end{array}$} & \multirow[b]{2}{*}{ sustrato } & \multirow[b]{2}{*}{ temporalidad } & \multirow[b]{2}{*}{ Tipología } & \multirow{2}{*}{$\begin{array}{r}\text { origen } \\
\text { agua }\end{array}$} \\
\hline & & & $\mathbf{X}$ & $\mathbf{Y}$ & & & & & & & & \\
\hline 1 & Desembocadura Río Yali & $22 / 07 / 2008$ & 245647 & 6260122 & 5 & 488.4 & 352.7 & & arena negra & $\mathrm{P}$ & $\mathrm{E}$ & $\mathrm{C}-\mathrm{M}$ \\
\hline 2 & Laguna Costera Yali-1 & $22 / 07 / 2008$ & 245860 & 6260486 & 6 & 232.9 & 165.1 & 2.947 .8 & arena & $\mathrm{P}$ & $\mathrm{LC}$ & $\mathrm{M}-\mathrm{C}$ \\
\hline 3 & Laguna Costera Yali-2 & $22 / 07 / 2008$ & 247734 & 6261567 & 5 & 223.9 & 165.1 & 2.947 .8 & arena compactada & $\mathrm{P}$ & $\mathrm{LC}$ & $\mathrm{M}-\mathrm{C}$ \\
\hline 4 & Laguna Colejuda & $22 / 07 / 2008$ & 249813 & 6260991 & 7 & 2.328 .4 & 1.261 .9 & 3.148 .1 & arcilla, sedimentos & $\mathrm{P}$ & $\mathrm{L}$ & $\mathrm{Sp}-\mathrm{Sb}$ \\
\hline 5 & Laguna Matanzas & $22 / 07 / 2008$ & 250374 & 6261470 & 34 & 3.014 .1 & 754.4 & 657.3 & arcilla, sedimentos & $\mathrm{P}$ & $\mathrm{L}$ & $\mathrm{C}$ \\
\hline 6 & Tranque los Molles & $23 / 07 / 2008$ & 250482 & 6256423 & 18 & 5.463 .1 & 1.263 .3 & 2.511 .7 & arcilla & $\mathrm{P}$ & EM & $\mathrm{Sp}-\mathrm{Sb}$ \\
\hline \multirow[t]{2}{*}{7} & Debajo Tranque & & & & & & & & & & & \\
\hline & de Molles & $23 / 07 / 2008$ & 250449 & 6256318 & 16 & 6.194 .3 & 226.9 & 286.1 & arena & $\mathrm{T}$ & LI & $\mathrm{C}$ \\
\hline 8 & Salinas & $23 / 07 / 2008$ & 249428 & 6256774 & 7 & 5.248 .3 & 736.3 & 1.372 .5 & arena, restos de conchas & $\mathrm{T}$ & $\mathrm{S}$ & $\mathrm{Cl}$ \\
\hline \multirow[t]{2}{*}{9} & Yali aguas arriba & & & & & & & & & & & \\
\hline & de las Salinas & $23 / 07 / 2008$ & 247618 & 6256577 & 12 & 4.517 .7 & 55.6 & & arena, restos orgánicos & $\mathrm{P}$ & $\mathrm{R}$ & $\mathrm{Cl}$ \\
\hline 10 & Laguna Las Salinas & $23 / 07 / 2008$ & 248306 & 6257011 & 10 & 5.606 .7 & 272.2 & 674.5 & arena-grava & $\mathrm{P}$ & MR & $\mathrm{C}$ \\
\hline 11 & Laguna Seca & $23 / 07 / 2008$ & 248889 & 6258499 & 7 & 3.673 .1 & 248.6 & 436.1 & arcilla & $\mathrm{T}$ & $\mathrm{L}$ & $\mathrm{C}$ \\
\hline 12 & Laguna Guairao & $23 / 07 / 2008$ & 249207 & 6259972 & 40 & 2.496 .3 & 110.6 & 355.5 & arena-grava & $\mathrm{P}$ & $\mathrm{L}$ & $\mathrm{C}$ \\
\hline 13 & Laguna Cabildo & $24 / 07 / 2008$ & 251550 & 6261929 & 29 & 2.255 .6 & 651.9 & 1.196 .3 & arena-grava & $\mathrm{P}$ & $\mathrm{L}$ & $\mathrm{Sb}-\mathrm{Sp}$ \\
\hline 14 & Laguna del Rey & $24 / 07 / 2008$ & 245584 & 6256515 & 9 & 4.044 .9 & 924.6 & 2.127 .3 & arena-grava & $\mathrm{P}$ & $\mathrm{L}$ & $\mathrm{Sb}-\mathrm{Sp}$ \\
\hline 15 & Puente Yali 2 & $25 / 07 / 2008$ & 255307 & 6253288 & 20 & 11.363 .2 & 3.2 & & arena, grava, restos orgánicos & $\mathrm{P}$ & $\mathrm{R}$ & $\mathrm{C}$ \\
\hline 16 & Estero Maintenlahue & $25 / 07 / 2008$ & 248471 & 6244869 & 52 & 10.041 .3 & 7.9 & & & $\mathrm{P}$ & $\mathrm{R}$ & $\mathrm{C}$ \\
\hline 17 & Mostazal desembocadura & $25 / 07 / 2008$ & 239744 & 6252374 & 9 & 270.9 & 90.1 & & arena, arcilla, grava & $\mathrm{P}$ & $\mathrm{E}$ & $\mathrm{C}-\mathrm{M}$ \\
\hline 18 & Tranque frente Bucalemito & $25 / 07 / 2008$ & 241272 & 6246666 & 93 & 2.730 .7 & 33.6 & 150.2 & arcilla & $\mathrm{P}$ & EM & $\mathrm{C}$ \\
\hline 19 & Laguna Costera Trihue & $27 / 07 / 2008$ & 252973 & 6266238 & 8 & 146.6 & 13.6 & 184.9 & arena negra & $\mathrm{P}$ & $\mathrm{LC}$ & M-C \\
\hline \multirow[t]{2}{*}{20} & Desembocadura & & & & & & & & & & & \\
\hline & Estero Peumo & $27 / 07 / 2008$ & 253107 & 6266576 & 7 & 39.0 & 23.9 & & cantos-grava & $\mathrm{P}$ & A & $\mathrm{C}$ \\
\hline
\end{tabular}

$\mathrm{P}=$ permanente; $\mathrm{T}=$ temporal. $\mathrm{E}=$ estuario, $\mathrm{LC}=$ laguna costera, $\mathrm{L}=$ laguna, $\mathrm{EM}=$ embalse, $\mathrm{LI}=$ llanura de inundación, $\mathrm{S}=$ salinas, $\mathrm{MR}=$ meandro del río, $\mathrm{R}=$ río, $\mathrm{A}=$ arroyo $\mathrm{C}-\mathrm{M}=$ continental-marino, $\mathrm{M}-\mathrm{C}=$ marino-continental, $\mathrm{Sp}-\mathrm{Sb}=$ superficial-subterráneo, $\mathrm{C}=$ continental, $\mathrm{Sb}-\mathrm{Sp}=$ subterráneo-superficial . 
ductivímetro Cole Parmer Mod. 19820) y oxígeno disuelto (mg/l, oxigenómetro Hanna HI 9142).

Posteriormente, se procedía a la recogida de las muestras de agua en botellas de polietileno, previamente lavadas con ácido, para analizar el resto de variables físico-químicas. Una parte de la muestra se reservaba para la medida de la alcalinidad (mg/l) (método de Gran; Mackereth et al., 1978), que se realizaba al final del día. Con el fin de no utilizar conservantes que pudieran alterar los resultados, al finalizar el día, las muestras recogidas eran enviadas por autobús al laboratorio del Centro EULA, donde inmediatamente se procesaban, de tal manera que nunca transcurrieron más de 24 horas desde la recogida de las mismas hasta su procesado.

Las variables medidas fueron: calcio, potasio, magnesio, cloruros, sulfatos, nitratos, nitritos, amonio, nitrógeno total y SRP. Todas las variables se expresaron en $\mathrm{mg} / \mathrm{l}$. Los métodos utilizados son los propuestos por el Standard Methods (APHA, 1985).

Con el fin de testar las posibles relaciones entre las variables físico-químicas medidas y distintas variables geográficas, se midieron la altitud sobre el nivel del mar (GPS), anchura del cuerpo de agua y distancia al mar (fotografía aérea) de todos los puntos de muestreo.

\section{Análisis de los datos}

Para establecer las relaciones aniónicas y catiónicas pertinentes, y elaborar los diagramas de $\mathrm{Pi}$ per (Piper, 1944), los datos de aniones y cationes expresados en $\mathrm{mg} / \mathrm{l}$ fueron transformados a meq/l. La concentración de sodio se dedujo de la siguiente ecuación: $\mathrm{CO}_{3}^{=}+\mathrm{SO}_{4}^{=}+\mathrm{Cl}^{-}=\mathrm{Na}^{+}+\mathrm{K}^{+}$ $+\mathrm{Ca}^{++}+\mathrm{Mg}^{++}$. Igualmente, se calculó el error del balance iónico según la ecuación (Custodio $\&$ Llamas, 1983): error $(\%)=200$ ( $\sum$ cationes $\sum$ aniones $/ \sum$ cationes $+\sum$ aniones). En general, el error admitido debe ser inferior al $15 \%$, aunque en la práctica se admiten errores algo superiores (Custodio \& Llamas, 1983). En nuestro caso, únicamente la Laguna Seca (11) presenta un error superior a este valor $(18 \%)$.

Para el análisis conjunto de los datos se calcularon las correlaciones de Pearson y se aplicó un análisis de componentes principales (PCA) sobre la matriz de datos transformada $\log (x+1)$. Para la interpretación de los resultados de PCA se realizó una clasificación mediante un análisis de conglomerados utilizando los programas SPSS 16 y Primer v6.1.2. (Clarke \& Gorley, 2006).

\section{RESULTADOS}

\section{Características ambientales}

Los humedales y cuerpos de agua prospectados se encuentran a una altitud muy escasa, entre 5 m.s.n.m. en la laguna costera Yali $(1$ y 3$)$ y 92 m.s.n.m. en el tranque frente a Bucalemito (18). Su distancia al mar oscila entre $39 \mathrm{~m}$ en la desembocadura del Estero Peumo (20) y $11363 \mathrm{~m}$ en el río Yali (15).

Dado que se trata de cuerpos de agua de diferentes tipologías (Tabla 1), sus dimensiones también difieren. Destacan por su tamaño, las lagunas Matanzas (5) y del Rey (14) y el tranque de Molles (6). En cuanto al sustrato, dominan los materiales finos (arenas, fundamentalmente, y arcilla). Hay que destacar el sustrato de arenas donde se mezclan restos de conchas en las salinas del Yali (8), que denotan el origen marino de esta cubeta. Por otro lado se detectaron abundantes restos orgánicos en las lagunas Colejuda (4), Matanzas (5), y en el río Yali (9 y 15).

En relación con la temporalidad del agua, todos los cuerpos muestreados, a excepción de la laguna Seca (11), aguas abajo del tranque de Molles (7) y las salinas (8), son permanentes.

En cuanto a la vegetación acuática y ribereña dominante, buena parte de los cuerpos de agua muestreados presentan prados extensos de pastizal utilizados por el ganado. En las riberas son habituales las mezclas entre el saladar, juncal y pajonales (Typha sp.). Aún quedan restos de bosque donde domina el arbusto nativo molle (Schinus latifolius), que se localiza preferentemente en la región central de Chile. Dentro de las formaciones de saladar hay que destacar a Sarcocornia fruticosa y distintas especies de Limonium y, dentro de los juncales, a Scirpus californicus. Gran parte de los cuerpos de agua muestreados 
tienen sus orillas cubiertas por plantaciones de eucaliptos (Eucalyptus globulus Labill). Finalmente, en el río Yali (15) existe abundante presencia de aromo (Acacia sp.) sustituyendo prácticamente al bosque ribereño nativo. En cuanto a los macrófitos, Ceratophyllum chilensis, se localiza únicamente en el tranque Los Molles (6) y Ranunculus sp. en la Laguna Guairao (12). Hay que destacar la presencia de densas formaciones de la cianofícea Anabaena encontradas en las lagunas Matanzas (5) y Cabildo (13).

\section{Hidroquímica del agua}

El valor medio de la temperatura del agua fue de $13.45^{\circ} \mathrm{C}$ y para el $\mathrm{pH}$, de 7.9. En ambos casos la desviación estándar fue muy baja. No ocurre igual con la conductividad que, aunque presenta un valor medio de $4660 \mu \mathrm{S} / \mathrm{cm}$, los valores máximo y mínimo fueron de $58800 \mu \mathrm{S} / \mathrm{cm}$ en la laguna costera el Yali (2) y $320 \mu \mathrm{S} / \mathrm{cm}$ en la desembocadura del Estero Peumo (20), respectivamente.

El valor medio del oxígeno disuelto fue de $9.8 \mathrm{mg} / \mathrm{l}$, con un rango entre $19.6 \mathrm{mg} / \mathrm{l}$ en la laguna Cabildo (13) y $3 \mathrm{mg} / \mathrm{l}$ en la de Guairao (12). La alcalinidad osciló entre $39.3 \mathrm{mg} / \mathrm{l}$ en la laguna Cabildo (13) y $6.2 \mathrm{mg} / \mathrm{l}$ en el río Yali (15), con un valor medio de $13.6 \mathrm{mg} / \mathrm{l}$.

En cuanto a los cationes, el valor medio más alto fue para el sodio $(521.17 \mathrm{mg} / \mathrm{l})$, seguido del magnesio $(72.6 \mathrm{mg} / \mathrm{l})$, potasio $(24 \mathrm{mg} / \mathrm{l})$ y calcio $(21.7 \mathrm{mg} / \mathrm{l})$. El rango de variación más importante es para el sodio, con valores extremos de $10856.5 \mathrm{mg} / \mathrm{l}$ en la laguna costera el Yali (2) y $4.49 \mathrm{mg} / \mathrm{l}$ en el tranque, frente a Bucalemito (18). En relación a los aniones, el cloruro es claramente dominante, con un valor medio de $963.85 \mathrm{mg} / \mathrm{l}$, frente al valor de $88.3 \mathrm{mg} / \mathrm{l}$ para el sulfato. Sin embargo, los cloruros presentan un rango mucho más amplio, entre $19163 \mathrm{mg} / \mathrm{l}$ en la laguna costera el Yali (2) y $32.6 \mathrm{mg} / \mathrm{l}$ en el río Yali (15).

En cuanto a los nutrientes, en todos los casos la desviación estándar es baja. Los valores medios para el nitrógeno total y sus fracciones son: $4.7 \mathrm{mg} / \mathrm{l} \mathrm{NT} ; 1.9 \mathrm{mg} / \mathrm{l}$ nitratos; $0.05 \mathrm{mg} / \mathrm{l}$ nitritos y $0.25 \mathrm{mg} / \mathrm{l}$ amonio. Hay que destacar, sin embargo, los altos valores de nitrógeno total detectados en las lagunas de Cabildo (13) (55.73 mg/l) y del Rey (14) $(14.55 \mathrm{mg} / \mathrm{l})$ y de nitratos en el estero Maitenlahue (16) $(19.6 \mathrm{mg} / \mathrm{l})$ y en la laguna del Mostazal (17) $(14.86 \mathrm{mg} / \mathrm{l})$. El valor medio de SRP fue de $0.4 \mathrm{mg} / \mathrm{l}$, con un rango entre $2.67 \mathrm{mg} / \mathrm{l} \mathrm{en} \mathrm{la} \mathrm{Laguna} \mathrm{Seca} \mathrm{(11)} \mathrm{y} 0.012 \mathrm{mg} / \mathrm{l}$ en las lagunas Cabildo (13) y costera Trihue (19).

En la Tabla 2 se presentan las correlaciones de Pearson calculadas para las variables geográficas y físico-químicas analizadas. Aquellas que mostraron correlaciones significativas $(p<0,05)$ son la conductividad con todos los aniones y cationes, así como con la anchura del

Tabla 2. Correlaciones de Pearson para todas las variables físico-químicas medidas en los humedales prospectados en el complejo El Yali. (* $p<0.05 ; * *<0.01)$. Pearson's correlations for physical and physical-chemical variables measured in the studied wetlands in the Yali wetlands complex. $(* \mathrm{p}<0.05 ; * * \mathrm{p}<0.01)$.

\begin{tabular}{|c|c|c|c|c|c|c|c|c|c|c|c|c|c|c|c|c|c|}
\hline & $\begin{array}{c}\text { Anchura } \\
(\mathrm{m})\end{array}$ & $\begin{array}{c}\text { Distancia } \\
\operatorname{mar}(\mathrm{m})\end{array}$ & $\begin{array}{l}\text { Altitud } \\
(\mathrm{m})\end{array}$ & $\begin{array}{c}\mathrm{T}^{\mathrm{a}} \text { agua } \\
\left({ }^{\circ} \mathrm{C}\right)\end{array}$ & $\mathrm{pH}$ & $\begin{array}{l}\text { Conduct. } \\
(\mu \mathrm{S} / \mathrm{cm})\end{array}$ & $\begin{array}{c}\mathrm{O}_{2} \\
(\mathrm{mg} / \mathrm{l})\end{array}$ & $\begin{array}{c}\text { Alcalinidad } \\
(\mathrm{mg} / \mathrm{l})\end{array}$ & $\begin{array}{c}\mathrm{NH}_{4}^{+} \\
(\mathrm{mg} / \mathrm{l})\end{array}$ & $\begin{array}{l}\mathrm{Ca}^{2+} \\
(\mathrm{mg} / \mathrm{l})\end{array}$ & $\begin{array}{c}\mathrm{Cl}^{-} \\
(\mathrm{mg} / \mathrm{l})\end{array}$ & $\begin{array}{l}\mathrm{Mg}^{2+} \\
(\mathrm{mg} / \mathrm{l})\end{array}$ & $\begin{array}{l}\mathrm{NO}_{3}^{-} \\
(\mathrm{mg} / \mathrm{l})\end{array}$ & $\begin{array}{l}\mathrm{NO}_{2}^{-} \\
(\mathrm{mg} / \mathrm{l})\end{array}$ & $\begin{array}{cl}\text { N.T. } & \mathrm{PO}_{4}^{3-} \\
(\mathrm{mg} / \mathrm{l}) & (\mathrm{mg} / \mathrm{l})\end{array}$ & $\begin{array}{l}\mathrm{K}^{+} \\
(\mathrm{mg} / \mathrm{l})\end{array}$ & $\begin{array}{l}\mathrm{SO}_{4}^{2-} \\
(\mathrm{mg} / \mathrm{l})\end{array}$ \\
\hline Distancia & 0.14 & & & & & & & & & & & & & & & & \\
\hline Altitud & -0.26 & $0.50^{*}$ & & & & & & & & & & & & & & & \\
\hline $\mathrm{T}^{o}$ & $0.59^{* *}$ & -0.13 & -0.03 & & & & & & & & & & & & & & \\
\hline $\mathrm{pH}$ & $0.51 *$ & -0.13 & -0.17 & 0.28 & & & & & & & & & & & & & \\
\hline Conduct. & $0.62 * *$ & -0.15 & $-0.47 *$ & 0.33 & 0.57 ** & & & & & & & & & & & & \\
\hline $\mathrm{O} 2$ & -0.19 & -0.42 & -0.18 & -0.32 & 0.34 & 0.18 & & & & & & & & & & & \\
\hline Alcalinidad & $0.69 * *$ & -0.08 & 0.01 & $0.62 * *$ & $0.71^{* *}$ & 0.37 & 0.01 & & & & & & & & & & \\
\hline $\mathrm{NH}_{4}^{+}$ & -0.20 & 0.24 & 0.09 & -0.10 & $-0.69 * *$ & -0.44 & $-0.49^{*}$ & -0.38 & & & & & & & & & \\
\hline $\mathrm{Ca}^{2+}$ & $0.51^{*}$ & -0.35 & $-0.61 * *$ & $=0.35$ & 0.38 & $0.87^{* *}$ & 0.16 & 0.26 & -0.26 & & & & & & & & \\
\hline $\mathrm{Cl}^{-}$ & $0.60^{* * *}$ & -0.35 & $-0.54^{*}$ & 0.32 & $0.55^{*}$ & $0.94 * *$ & 0.23 & 0.37 & -0.40 & $0.91 * *$ & & & & & & & \\
\hline $\mathrm{Mg}^{2+}$ & $0.54 *$ & -0.36 & -0.43 & 0.39 & $0.45^{*}$ & $0.92 * *$ & 0.20 & 0.37 & -0.40 & $0.90^{* *}$ & $0.97 * *$ & & & & & & \\
\hline $\mathrm{NO}_{3}^{-}$ & $-0.52 *$ & -0.02 & 0.14 & -0.28 & $-0.86 * *$ & $-0.48^{*}$ & -0.09 & $-0.64^{* *}$ & $0.62^{* *}$ & -0.23 & -0.42 & -0.36 & & & & & \\
\hline $\mathrm{NO}_{2}^{-}$ & $-0.47 *$ & 0.17 & 0.36 & -0.17 & $-0.68 * *$ & -0.44 & -0.00 & $-0.63^{* *}$ & 0.43 & -0.28 & -0.44 & -0.39 & $0.85^{* * *}$ & & & & \\
\hline N.T. & 0.26 & 0.39 & $0.51^{*}$ & 0.39 & 0.10 & -0.20 & -0.23 & $0.50^{*}$ & 0.29 & -0.29 & -0.25 & -0.25 & 0.03 & 0.15 & & & \\
\hline $\mathrm{PO}_{4}^{3-}$ & -0.28 & 0.31 & 0.08 & -0.03 & $-0.82 * *$ & $-0.47 *$ & $-0.50^{*}$ & $-0.64^{* * *}$ & $0.69^{* *}$ & -0.30 & $-0.52 *$ & $-0.47^{*}$ & $0.62 * *$ & $0.64 * *$ & -0.04 & & \\
\hline $\mathrm{K}^{+}$ & 0.44 & -0.40 & -0.38 & $0.44^{*}$ & 0.40 & $0.86^{* *}$ & 0.20 & 0.26 & -0.32 & $0.86^{* *}$ & $0.89^{* *}$ & $0.92 * *$ & -0.26 & -0.21 & $-0.24-0.32$ & & \\
\hline $\mathrm{SO}_{4}^{2-}$ & 0.36 & -0.32 & $-0.62^{* * *}$ & 0.33 & 0.13 & $0.74 * *$ & -0.09 & -0.01 & -0.04 & $0.84 * *$ & $0.78^{* * *}$ & $0.77^{* * *}$ & -0.13 & -0.17 & $-0.37 \quad 0.01$ & 0.77 ** & \\
\hline $\mathrm{Na}^{+}$ & $0.61^{* *}$ & -0.34 & $-0.65^{* *}$ & 0.29 & $0.59 * *$ & $0.92 * *$ & 0.24 & 0.39 & -0.37 & $0.93 * *$ & $0.97^{* *}$ & $0.90^{* * *}$ & -0.43 & $-0.47 *$ & $-0.24-0.51 *$ & $0.83^{* *}$ & $0.77^{* *}$ \\
\hline
\end{tabular}


cuerpo de agua (correlación positiva) y con al altitud (correlación negativa). Esta situación se observa también para la mayor parte de aniones $\mathrm{y}$ cationes que presentan correlaciones positivas con la anchura y negativas con la altitud. La temperatura del agua presenta correlaciones positivas con la anchura del cuerpo de agua y la alcalinidad. El oxígeno disuelto presenta una correlación negativa con el amonio, denunciando procesos de descomposición anaerobia. El pH mantiene correlaciones positivas con algunos iones pero negativas con todos los nutrientes. En cuanto a los nutrientes, todos ellos mantienen correlaciones positivas entre si, a excepción del nitrógeno total.

\section{Contenido salino y composición iónica}

En relación con la mineralización y el contenido salino de las aguas se observa una situación compleja. Los puntos de muestreo se clasificaron en cuatro grupos según los valores de conductividad: dulces $(<1500 \mu \mathrm{S} / \mathrm{cm})$, subsalinas (1500$5000 \mu \mathrm{S} / \mathrm{cm})$, hiposalinas $(5000-35000 \mu \mathrm{S} / \mathrm{cm})$ y mesosalinas (35 000-70 $000 \mu \mathrm{S} / \mathrm{cm})$. Las aguas dulces se corresponden con cuerpos localizados principalmente en esteros $(15,16,17,20)$ en el tranque frente a Bucalemito (18), y en dos lagunas, Seca (11) y Guairao (12). Las subsalinas con las lagunas Matanzas (5) y Salinas (10), debajo del tranque de Molles (7) y con el tramo del río Yali antes de su desembocadura (9). Las hiposalinas, con las lagunas Cabildo (13) y Rey (14), las costeras Yali (3) y Trihue (19), el tranque de Molles (6) y con las salinas en la llanura del Yali (8) y mesosalinas, con la desembocadura del río Yali (1), la laguna costera Yali (2) y con la laguna Colejuda (4).

Espacialmente, constituye un gradiente desde ambos lados del complejo de humedales, donde se detectan los valores más bajos de conductividad, hacia el centro y en dirección al mar donde, en forma de gradiente, aumentan los valores de conductividad, con una anomalía interior formada por las lagunas Seca y Guairao (11 y 12) Fig. 1).

En la Tabla 3 se presentan los estadísticos más importantes de los puntos de muestreo según su tipología salina y en la figura 2 , los diagramas de Piper que permiten representar de forma simultánea aniones y cationes. En todos los casos

Tabla 3. Principales estadísticos de los grupos de puntos de muestreo del complejo El Yali según su salinidad. (SD = desviación estándar; CV = coeficiente de variación). Main statistical parameters for the sampling sites in the Yali wetlands complex according to their salinity $(S D=$ standard deviation, $C V=$ Coefficient of variation $)$.

\begin{tabular}{|c|c|c|c|c|c|c|c|c|}
\hline & & $\begin{array}{l}\text { Alcalinidad } \\
\text { (meq/1) }\end{array}$ & $\begin{array}{c}\mathrm{Cl}^{-} \\
(\mathrm{meq} / \mathrm{l})\end{array}$ & $\begin{array}{c}\mathrm{SO}_{4}^{=} \\
(\mathrm{meq} / \mathrm{l})\end{array}$ & $\begin{array}{c}\mathrm{Ca}^{2+} \\
(\mathrm{meq} / \mathrm{l})\end{array}$ & $\begin{array}{c}\mathrm{Mg}^{2+} \\
(\mathrm{meq} / \mathrm{l})\end{array}$ & $\begin{array}{c}\mathrm{Na}^{+} \\
(\mathrm{meq} / \mathrm{l})\end{array}$ & $\begin{array}{c}\mathrm{K}^{+} \\
(\mathrm{meq} / \mathrm{l})\end{array}$ \\
\hline \multirow{5}{*}{$\begin{array}{c}\text { Aguas dulces } \\
(<1500 \mu \mathrm{S} / \mathrm{cm}) \\
n=7\end{array}$} & Media & 0.42 & 3.45 & 0.92 & 0.45 & 1.80 & 2.17 & 0.41 \\
\hline & Max & 0.66 & 8.93 & 3.02 & 1.32 & 3.36 & 6.22 & 0.90 \\
\hline & Min & 0.21 & 0.92 & 0.25 & 0.08 & 0.64 & 0.20 & 0.12 \\
\hline & $\mathrm{SD}$ & 0.18 & 2.76 & 0.97 & 0.43 & 1.27 & 2.19 & 0.26 \\
\hline & $\mathrm{CV}(\%)$ & 43.82 & 79.92 & 105.10 & 93.83 & 70.52 & 100.86 & 63.24 \\
\hline \multirow{5}{*}{$\begin{array}{c}\text { Aguas subsalinas } \\
(1500-5000 \mu \mathrm{S} / \mathrm{cm}) \\
n=4\end{array}$} & Media & 0.56 & 22.86 & 1.67 & 0.85 & 5.84 & 17.96 & 0.49 \\
\hline & Max & 1.20 & 33.70 & 3.61 & 1.27 & 9.17 & 29.56 & 0.67 \\
\hline & Min & 0.27 & 17.37 & 0.03 & 0.59 & 2.94 & 11.36 & 0.33 \\
\hline & SD & 0.44 & 7.36 & 1.49 & 0.33 & 2.66 & 7.98 & 0.14 \\
\hline & $\mathrm{CV}(\%)$ & 77.68 & 32.19 & 89.17 & 38.73 & 45.56 & 44.44 & 28.18 \\
\hline \multirow{5}{*}{$\begin{array}{c}\text { Aguas hiposalinas } \\
(5000-35000 \mu \mathrm{S} / \mathrm{cm}) \\
n=6\end{array}$} & Media & 0.69 & 148.05 & 17.23 & 4.25 & 30.58 & 129.41 & 2.61 \\
\hline & Max & 1.31 & 492.25 & 48.58 & 12.23 & 93.91 & 429.30 & 9.23 \\
\hline & Min & 0.39 & 36.04 & 1.21 & 1.06 & 5.91 & 31.15 & 0.56 \\
\hline & $\mathrm{SD}$ & 0.35 & 174.94 & 20.89 & 4.26 & 33.20 & 152.15 & 3.32 \\
\hline & $\mathrm{CV}(\%)$ & 50.00 & 118.16 & 121.21 & 100.19 & 108.58 & 117.57 & 127.30 \\
\hline \multirow{5}{*}{$\begin{array}{c}\text { Aguas mesosalinas } \\
(35000-70000 \mu \mathrm{S} / \mathrm{cm}) \\
n=3\end{array}$} & Media & 0.50 & 435.21 & 38.43 & 9.50 & 77.38 & 382.13 & 7.85 \\
\hline & Max & 0.69 & 540.56 & 54.56 & 13.03 & 103.86 & 472.23 & 10.15 \\
\hline & Min & 0.39 & 369.87 & 22.48 & 3.16 & 30.56 & 318.71 & 4.77 \\
\hline & $\mathrm{SD}$ & 0.17 & 92.11 & 16.04 & 5.50 & 40.67 & 80.16 & 2.77 \\
\hline & $\mathrm{CV}(\%)$ & 34.33 & 21.17 & 41.73 & 57.92 & 52.55 & 20.98 & 35.35 \\
\hline
\end{tabular}



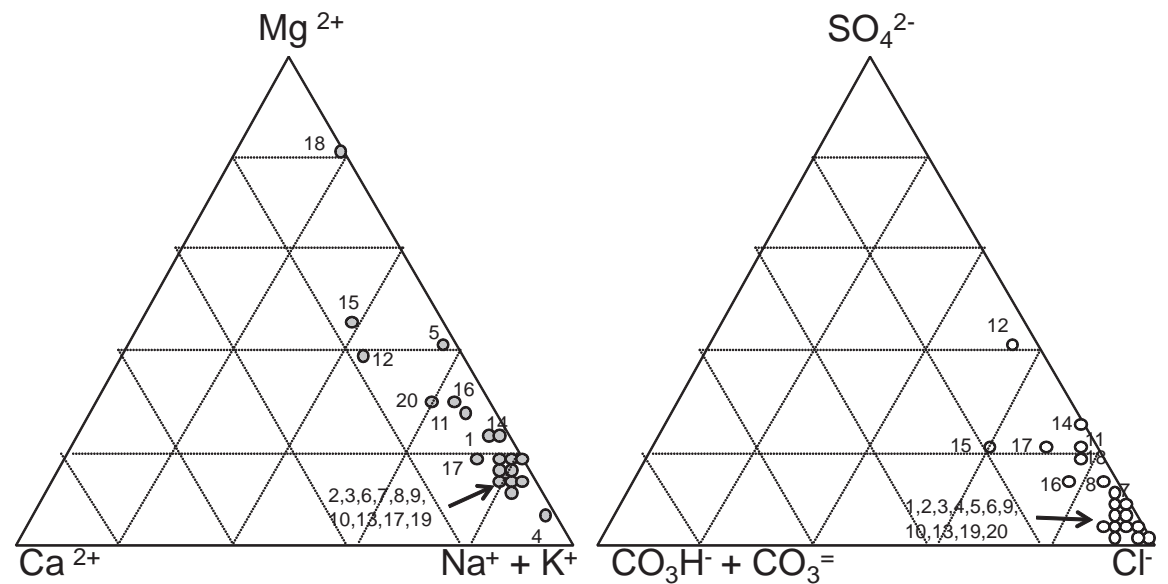

Figura 2. Diagramas de Piper para los puntos de muestreo del complejo El Yali. Piper diagrams for the sampling sites of the Yali wetlands complex.

la composición iónica está marcada por los cloruros y el sodio, observándose el gradiente salino desde las aguas dulces a las mesosalinas ya descrito. Sin embargo, cuando se analiza la secuencia aniónica y catiónica se aprecian algunas diferencias. En la mayoría de los puntos de muestreo la relación habitual es $\mathrm{Cl}^{-}>\mathrm{SO}_{4}^{=}>\mathrm{CO}_{3}^{=}$, salvo en las lagunas Cabildo (13), Matanzas (5) y Seca (11) y en el estero Maintenlahue (16), donde el carbonato es superior a los sulfatos.

En cuanto a la secuencia catiónica, el sodio es el dominante en todos los cuerpos de agua estudiados, a excepción del río Yali (15), estero Maitenlahue (16) y tranque frente a Bucalemito (18), donde lo es el magnesio. Hay cuatro secuencias diferentes, la más habitual es $\mathrm{Na}^{+}>\mathrm{Mg}^{2+}>\mathrm{Ca}^{2+}$ $>\mathrm{K}^{+}$, típica de aguas salinas. En las lagunas $\mathrm{Co}^{-}$ lejuda (4) y Matanzas (5) el potasio es mayor que el calcio $\left(\mathrm{Na}^{+}>\mathrm{Mg}^{2+}>\mathrm{K}^{+}>\mathrm{Ca}^{2+}\right)$. En los puntos donde el magnesio es dominante también aparecen dos secuencias diferentes: $\mathrm{Mg}^{2+}>\mathrm{Na}^{+}>$ $\mathrm{Ca}^{2+}>\mathrm{K}^{+}$en el río Yali (15) y estero Maitenlahue (16) y $\mathrm{Mg}^{2+}>\mathrm{K}^{+}>\mathrm{Na}^{+}>\mathrm{Ca}^{2+}$ en tranque frente a Bucalemito (18).

En la figura 3 se presentan los valores medios y error de tres índices iónicos $\left(\mathrm{SO}_{4}^{=} / \mathrm{CO}_{3}^{=}\right.$; $\mathrm{Cl}^{-} / \mathrm{CO}_{3}^{=}$y $\mathrm{Na}^{+} / \mathrm{Mg}^{2+}$ ) en los cuatro tipos de cuerpos de agua según su salinidad. Como se observa, todos ellos aumentan al hacerlo la salinidad, lo que indica la mayor importancia de los cloruros sobre el resto de aniones.
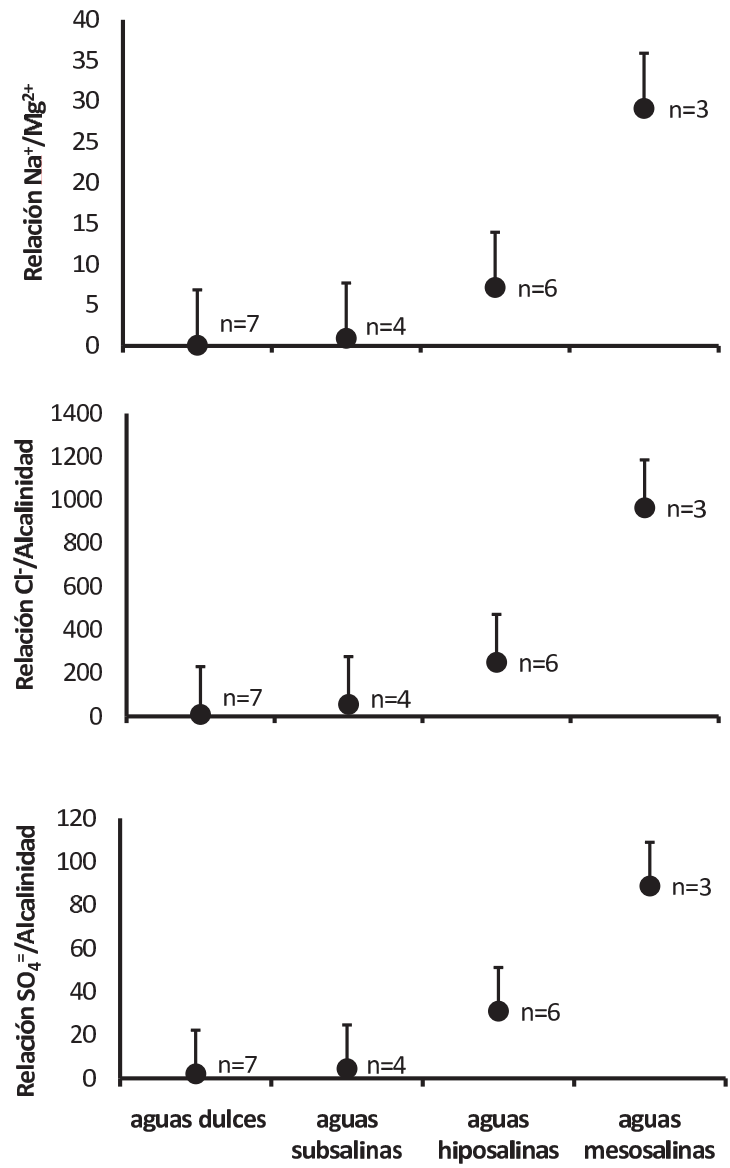

Figura 3. Valores medios de los tres índices iónicos para los cuatro tipos salinos detectados en los humedales del complejo El Yali. Average values of the three ionic indexes for the four saline types detected in the wetlands of the Yali wetlands complex. 


\section{Nutrientes y estado trófico}

En la figura 4a, se presenta la proporción de las fracciones orgánicas e inorgánicas de nitrógeno medidas en los cuerpos de agua muestreados. Prácticamente en la mitad de ellos la fracción orgánica supone más del $50 \%$ del total de nitrógeno. Todos ellos corresponden a las muestras de agua recogidas en lagunas, por el contrario, en todos los ríos, esteros y en la laguna costera Yali $(1,2,9,10,15,16,17$ y 20), la fracción inorgánica de nitrógeno supone el $100 \%$ del total.

En cuanto al nitrógeno inorgánico, el nitrito (Fig. 4b), es la fracción que menos contribuye al total. Se pueden diferenciar dos grupos de humedales: aquellos en los que la fracción dominante es el amonio (> 50\%) $(3,4,7,8,11,14,16,17 \mathrm{y}$ 19) y en los que domina el nitrato $(>50 \%)(1,5$, $6,9,10,12,13,15,18$ y 20).

En relación a las concentraciones de SRP, el valor más elevado $(2.67 \mathrm{mg} / \mathrm{l})$ se obtuvo en la laguna Seca (11) (Fig. 4c). De igual manera, se detectaron altas concentraciones en las lagunas Guairao (12), Mostazal (17) y en el tranque frente a Bucalemito (18), todas ellas influenciadas por actividades ganaderas.

La mayor parte de los cuerpos de agua muestreados, presentan una relación N/P inferior a 16, lo cual puede indicar una limitación de nitrógeno para los productores primarios (Fig. 4d) (Grimm \& Fisher, 1986). Los puntos de muestreo localizados en las lagunas Colejuda (4), Matanzas (5), Cabildo (13), del Rey (14), Mostazal (17), estero Maitenlahue (16) y desembocadura del estero Peumo (20) no presentan limitación de nitrógeno.

\section{Análisis multivariantes}

Con el fin de analizar la variación conjunta de todas las variables medidas (geográficas y físicoquímicas) y obtener grupos de estaciones de muestreo con un comportamiento similar, a la matriz de datos transformada se le aplicó un análisis de componentes principales (PCA). Los
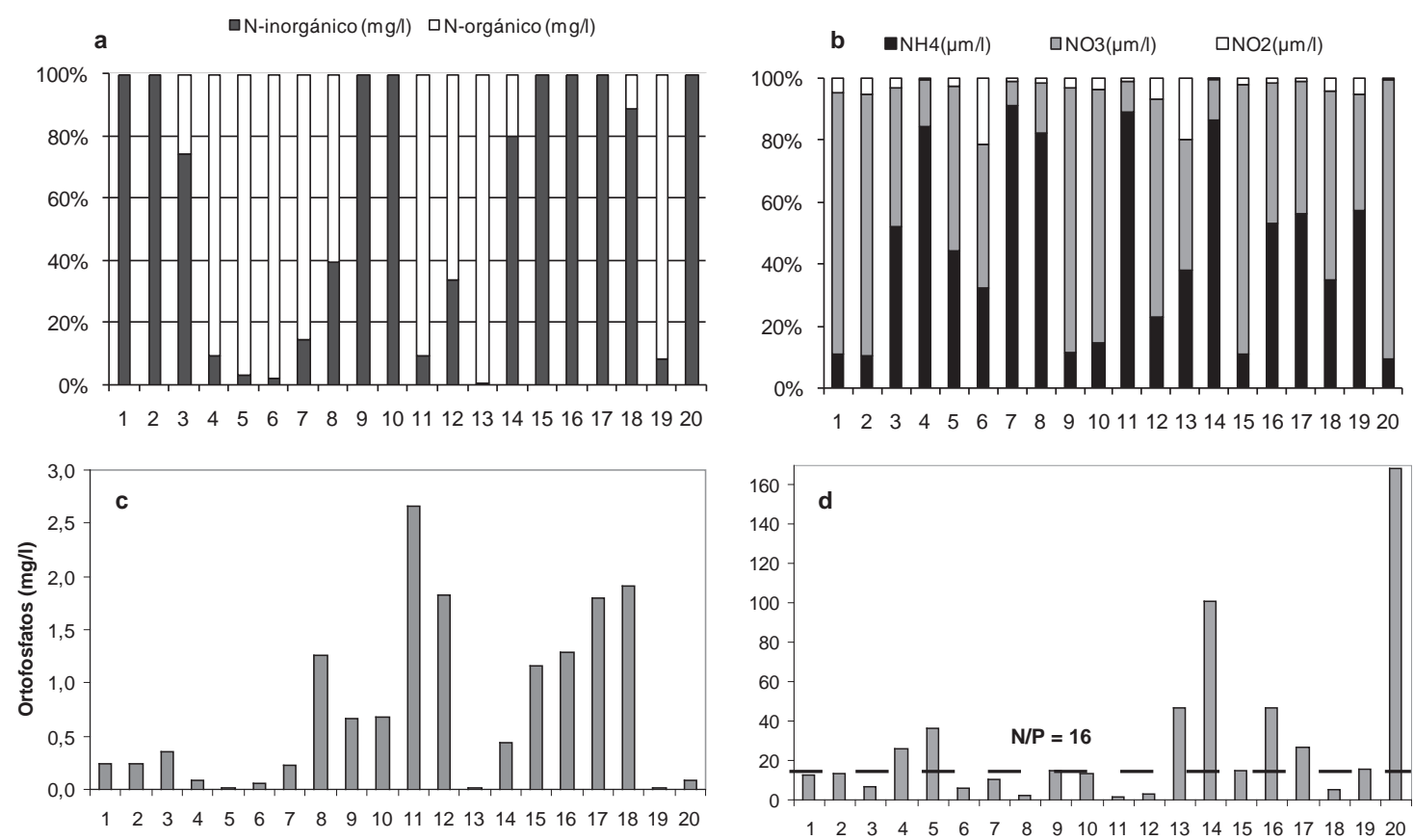

Figura 4. a) Proporción de las fracciones de nitrógeno orgánico e inorgánico, $b$ ) Proporción de las fracciones de nitrógeno inorgáni$\mathrm{co}, c)$ Concentración de SRP (mg/l) y d) Relación nitrógeno inorgánico/fósforo, medidos en los cuerpos de agua muestreados en el complejo El Yali. a) Percentage proportion of organic and inorganic nitrogen, b) Percentage proportions of inorganic nitrogen, c) Concentrations of SRP and d) Inorganic nitrogen/phosphorous relationship in the Yali wetlands complex. 
Tabla 4. Valores propios de las variables geográficas y físico-químicas del agua medidas para los tres primeros ejes del análisis de componentes principales (PCA) y varianza absorbida por cada uno de los ejes. Valores propios $<0.20$ fueron omitidos. Eigen-values of the geographical and physico-chemical variables measured for the first three components of PCA and the absorbed variance for each of them. Eigen-values $<0.20$ have been omitted.

\begin{tabular}{|c|c|c|c|}
\hline Variable & PC1 & PC2 & $\mathbf{P C 3}$ \\
\hline Anchura (m) & -0.223 & 0.595 & \\
\hline Distancia al mar (m) & & 0.648 & -0.550 \\
\hline Altitud (m) & & & \\
\hline $\mathrm{T}^{\mathrm{a}}$ agua $\left({ }^{\circ} \mathrm{C}\right)$ & & & \\
\hline $\mathrm{pH}$ & & & \\
\hline Conductividad $(\mu \mathrm{S} / \mathrm{cm})$ & -0.343 & & \\
\hline $\mathrm{O}_{2}(\mathrm{mg} / \mathrm{l})$ & & & \\
\hline Alcalinidad (mg/l) & & & 0.221 \\
\hline Amonio (mg/l) & & & -0.223 \\
\hline Calcio $(\mathrm{mg} / \mathrm{l})$ & -0.276 & & \\
\hline Cloruros (mg/l) & -0.429 & & \\
\hline Magnesio (mg/l) & -0.328 & & \\
\hline Nitrato $(\mathrm{mg} / \mathrm{l})$ & & -0.234 & -0.289 \\
\hline Nitrito $(\mathrm{mg} / \mathrm{l})$ & & & \\
\hline Nitrógeno total $(\mathrm{mg} / \mathrm{l})$ & & 0.228 & \\
\hline Ortofosfato $(\mathrm{mg} / \mathrm{l})$ & & & \\
\hline Potasio $(\mathrm{mg} / \mathrm{l})$ & -0.243 & & \\
\hline Sulfato (mg/l) & -0.355 & -0.213 & -0.604 \\
\hline Sodio $(\mathrm{mg} / \mathrm{l})$ & -0.485 & & \\
\hline \% Varianza adsorbida & 70.7 & 11.9 & 5.6 \\
\hline
\end{tabular}

tres primeros ejes del análisis explican el $88.2 \%$ de la varianza total (Tabla 4). El primer eje está definido de forma negativa por la conductividad, cloruros, magnesio, sulfatos y sodio, por lo que se trata de un eje de mineralización. El se- gundo eje, al estar definido de forma positiva por la anchura y la distancia al mar, hace referencia al tamaño del cuerpo de agua. El tercer eje, mezcla los nutrientes principales con algunas variables relativas a la salinidad. La interpretación de

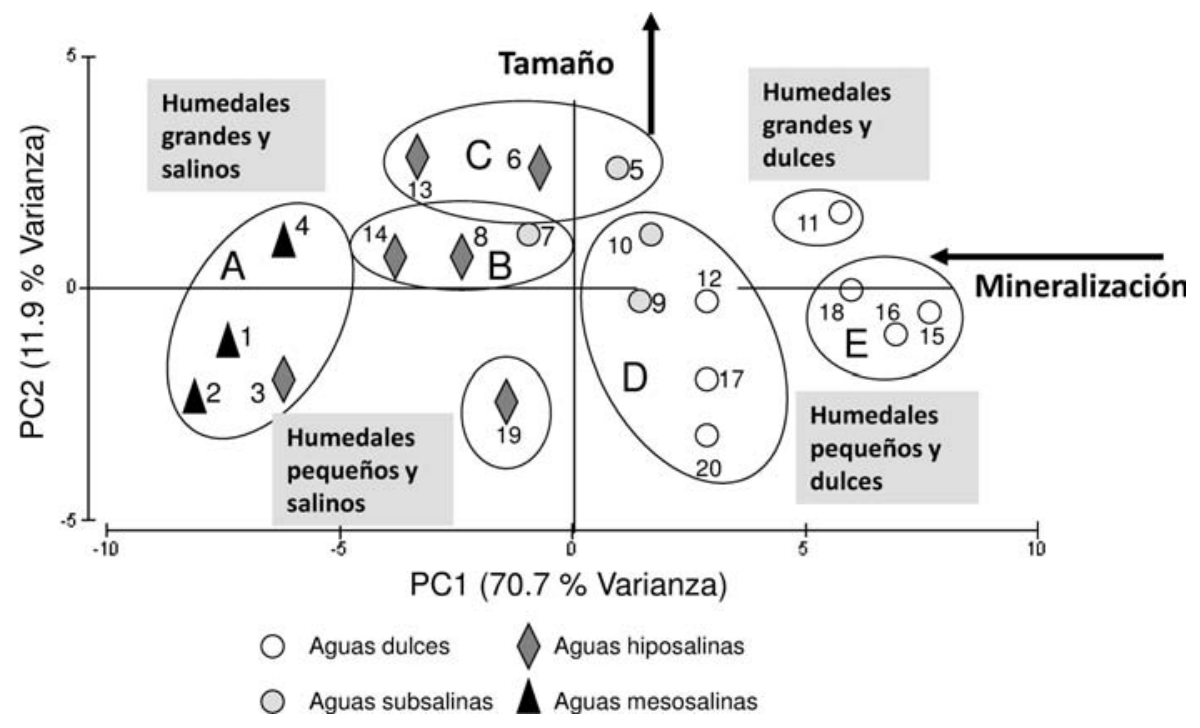

Figura 5. Proyección de los puntos de muestreo en el espacio definido por los dos primeros ejes del análisis de componentes principales (PCA). Sampling site projection in the space defined by the first two components of the Principal Component Analysis (PCA). 
los tres primeros componentes permite explicar el tipo de cuerpo de agua en cada cuadrante de la figura 5. Así, en el superior derecho se situarían los cuerpos de agua "dulces y grandes", en el inferior derecho, los "dulces y pequeños", en el superior izquierdo, los "salinos y grandes" y en el inferior izquierdo, los "salinos y pequeños".

Con el fin de ayudar a definir los grupos de estaciones con rasgos hidroquímicos similares, se realizó un análisis de clasificación ("cluster") utilizando distancias euclídeas, sobre las coordenadas de los tres primeros ejes de los puntos de muestreo obtenidas en el PCA (Fig. 6). El corte más ajustado permite definir 5 grupos de estaciones, quedando los puntos 11 y 19 (Lagunas Seca y Trihue) separados.

Cuando se llevan estos resultados a la proyección resultante del análisis de componentes principales se observan grupos que pueden ser identificados por sus características geográficas e hidroquímicas (Fig. 5). Así, el grupo A está constituido por cuatro estaciones, dos de ellas localizadas en la laguna costera Yali (2 y 3), la desembocadura de este río (1) y la laguna Colejuda (4).

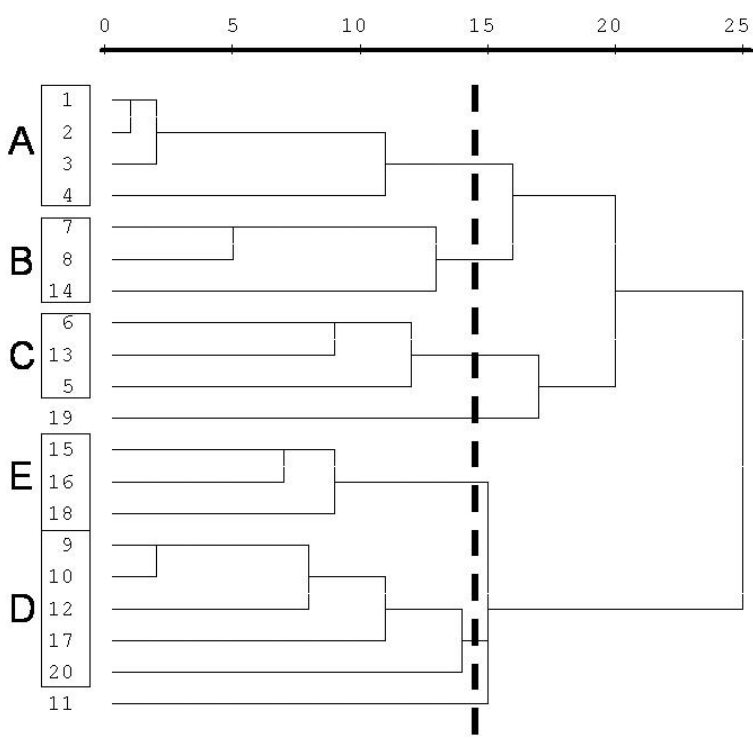

Figura 6. Dendrograma del análisis de clasificación realizado sobre las coordenadas de los tres primeros ejes del PCA. La línea discontinua señala el corte para el que se obtienen los grupos más coherentes. Dendrogram of the classification analysis performed on the coordinates of the first three PCA components. The dotted line shows the cut that obtained the most consistent groups.
Se caracterizan por presentar los valores más altos de salinidad, composición iónica típica de ambientes salinos por su cercanía al mar y por su tamaño relativamente grande. Los grupos B y C, aunque los separa el clúster, presentan rasgos hidroquímicos comunes. Incluye a las lagunas Matanzas (5), Cabildo (13) y Rey (14), el tranque los Molles (6), debajo del tranque (7) y las salinas (8). Se trata de cuerpos de agua hiposalinos y de tamaño grande. De estos grupos se separa la laguna costera Trihue (19) por su tamaño mucho más pequeño. El grupo D incluye prácticamente a todas las estaciones localizadas en las desembocaduras de ríos y esteros (9, 10, 12, 17 y 20). Se trata de aguas dulces o hiposalinas, de tamaño medio o pequeño. Por último, el grupo E, incluye a esteros (15 y 16) y el tranque frente a Bucalemito (18) de aguas muy dulces y tamaños pequeños. Se separa de estos grupos la laguna Seca (11) por su tamaño y temporalidad.

\section{DISCUSIÓN}

Es habitual que la seña de identidad e importancia de un humedal venga dada principalmente por su diversidad en aves $u$ otras comunidades biológicas, siendo escasos los trabajos que analizan las singularidades microbiológicas, hidrológicas o funcionales de estos ecosistemas. Este es el caso del humedal de El Yali, del que se conoce relativamente bien su fauna vertebrada (Leiva et al., 1995, Vilina \& López, 1996, Brito, 1999; Victoriano et al., 2006, CONAF, 2009), pero apenas existe información sobre otras comunidades biológicas (e.g. invertebrados acuáticos y terrestres, algas y macrófitos, etc.), y menos aún sobre sus particularidades hidrológicas, ciclos biogeoquímicos, sedimentología o hidroquímica.

Los resultados obtenidos en este estudio sobre distintas variables físico-químicas del agua medidas en 20 cuerpos de agua del complejo de humedales El Yali, revela la complejidad hidroquímica del sistema. En efecto, en un espacio relativamente pequeño $\left(115 \mathrm{~km}^{2}\right)$ conviven cuerpos de agua de muy distinta tipología, sobre todo en lo referente a su contenido salino. En una primera aproximación sobre el contenido salino de los hu- 
medales de El Yali (Fig. 1), se detecta un patrón general marcado por el aumento de la conductividad del agua en un gradiente en dirección oesteeste que se rompe en el centro, donde se registran los valores más bajos de salinidad (700 $\mu \mathrm{S} / \mathrm{cm}$ en la laguna Seca (11) y $1170 \mu \mathrm{S} / \mathrm{cm}$ en la laguna Guairao (12)). En el caso de la laguna Seca pueden explicarse por su temporalidad. El periodo de inundación de esta laguna está marcado, fundamentalmente, por las lluvias y la escorrentía que genera, de hecho permaneció seca durante todo el verano del 2008 (comunicación de lugareños). La laguna de Guairao, por el contrario, recibe vertidos líquidos de la industria avícola (valores altos de $\mathrm{SRP}=1.83 \mathrm{mg} / \mathrm{l}$ y bajos de oxígeno disuelto $=3.0 \mathrm{mg} / \mathrm{l})$ que, probablemente, dulcifica también el agua de la laguna.

Es interesante la alta correlación que mantiene la conductividad y prácticamente todos los aniones y cationes, de forma positiva con la anchura del cuerpo de agua y, de forma negativa con la altitud. En efecto, los puntos de muestreo situados a menor altitud son los más salinos y también son los cuerpos de agua más grandes. Sin embargo, no está relacionada con la distancia al mar, dado que algunas lagunas y cuerpos de agua más alejados del mar presentan, bien valores altos o más bajos de salinidad, independientemente de su distancia al mar. Así pues, esta variable no explica por sí sola la salinidad de estos cuerpos de agua. La topografía, el tipo desedimentos y sobre todo el origen del agua explican la dinámica hidrológica de los humedales (González-Bernáldez \& Rey Benayas, 1992; Kazezyilmaz-Alhan \& Medina, 2008).

En nuestro caso, no se disponen de estudios sobre los flujos de agua superficial, subsuperficial o subterránea que expliquen este patrón geoquímico, pero la interpretación de los datos obtenidos permite una aproximación al origen del agua en los humedales del complejo El Yali. Como se observa en los diagramas de Piper (Fig. 2), la mayor parte de los puntos de muestreo presentan una dominancia de cloruro y sodio, lo cual es lógico teniendo en cuenta que buena parte del territorio es una terraza marina (Borgel, 1983). Sin embargo, cuando se analizan las secuencias catiónicas, en tres de ellos $(15,16$ y 18) el magnesio domina sobre el sodio o el potasio, y se corres- ponden con dos esteros (El Yali y Maitenlahue) y el tranque frente a Bucalemito, una pequeña represa que recoge agua de escorrentía. En estos casos parece clara la dominancia de agua superficial. El sodio es el ión mas soluble y difícil de precipitar (Custodio \& Llamas, 1983), y puede tener distinta procedencia, la más habitual es la entrada de agua marina, pero también puede provenir de descargas de aguas subterráneas, en las que el tiempo de residencia incide sobre la sustitución del calcio o el magnesio por el sodio (Toth, 1984; Santos et al., 2008).

En cuanto a la secuencia aniónica, en la mayoría de los puntos de muestreo la relación habitual es $\mathrm{Cl}^{-}>\mathrm{SO}_{4}^{=}>\mathrm{CO}_{3}^{=}$. Esta secuencia se encuentra en todo tipo de agua, pero es más frecuente en cuencas endorreicas y en lagunas costeras (López, 1984). Son una excepción las lagunas Cabildo (13), Matanzas (5) y Seca (11) y el estero Maintenlahue (16), donde el carbonato es superior a los sulfatos, lo cual ocurre cuando el agua proviene de la escorrentía superficial (Santos et al., 2008) o en cuencas cerradas o de aguas poco mineralizadas (Alonso, 1998). En efecto, todas ellas, a excepción de la laguna Cabildo, están clasificadas como de aguas dulces o subsalinas. La laguna Matanzas es el final de una pequeña subcuenca que se podría considerar endorreica, dado que sólo cuando existe un superávit hídrico se produce un drenaje en dirección al mar. Los suelos de esta laguna parecen estar constituidos por estratos impermeables o semipermeables que la hacen presentar un comportamiento relativamente estable en cuanto a su nivel hídrico en periodos de sequía (Biblioredes, 2009), aunque no en cuanto a su hidroquímica. En efecto, esta laguna presenta oscilaciones importantes de salinidad, como lo demuestran los registros de conductividad medidos en dos años sucesivos en invierno $(1286 \mu \mathrm{S} / \mathrm{cm}$ en 2007 (Figueroa et al, 2009) y $4890 \mu \mathrm{S} / \mathrm{cm}$ en 2008). Esta situación también se produce en otras lagunas del complejo (Tabla 5). Las variaciones de salinidad del agua en las lagunas y humedales están marcadas por la intensidad de los procesos de evaporación y por la cantidad de lluvias del año hidrológico (Martino, 1988); sin embargo, en las lagunas de El Yali coexisten varias situaciones 
Tabla 5. Temperatura, $\mathrm{pH}$ y conductividad del agua medidos en invierno de 2007 y 2008 en algunas lagunas del complejo El Yali. (Los datos de 2007 se han extraído de Figueroa et al., 2009). Water temperature, pH and conductivity measured in the winters of 2007 and 2008 in some wetlands of the Yali wetland's complex. (The Data of 2007 have been removed from Figueroa et al., 2009).

\begin{tabular}{clrrrrrr}
\hline & & \multicolumn{2}{c}{$\mathrm{T}^{\mathrm{a}}$ agua $\left({ }^{\circ} \mathrm{C}\right)$} & \multicolumn{3}{c}{$\mathrm{pH}$} & \multicolumn{2}{c}{ Conductividad $(\mu \mathrm{S} / \mathrm{cm})$} \\
\cline { 3 - 7 } $\mathrm{N}^{\mathrm{N}}$ & nombre & 2007 & 2008 & 2007 & 2008 & 2007 & 2008 \\
\hline 4 & Laguna Colejuda & 11.6 & 14.6 & 8.4 & 8.72 & 52200 & 42700 \\
5 & Laguna Matanzas & 12.3 & 13.5 & 7.58 & 8.62 & 1286 & 4890 \\
6 & Tranque los Molles & 11.7 & 12.9 & 8.00 & 8.84 & 2160 & 12700 \\
7 & Debajo tranque de Molles & & 12.3 & & 8.40 & 4030 \\
10 & Laguna Las Salinas & 11.8 & 12.5 & 7.64 & 7.66 & 32100 & 2800 \\
11 & Laguna Seca & & 15.0 & & 7.82 & 700 \\
12 & Laguna Guairao & 10.9 & 17.8 & 7.30 & 7.79 & 1350 & 1170 \\
13 & Laguna Cabildo & 10.5 & 16.2 & 7.60 & 9.31 & 12360 & 14400 \\
14 & Laguna del Rey & 13.2 & 14.6 & 7.10 & 7.44 & 6780 & 15300 \\
\hline
\end{tabular}

diferentes (Tabla 5). Las lagunas Colejuda (4), Guairao (12), y Cabildo (13) no muestran una variación importante en los valores de conductividad, parecen más estables que las de Matanzas (5), Los Molles (6), y del Rey (14), que registraron valores muchos más altos de conductividad en invierno de 2008. La laguna de Las Salinas (10), por el contrario, registró valores mucho más bajos de conductividad. Esta situación no puede ser explicada por la climatología, dado que el año 2007 fue mucho más seco que el 2008, como lo demuestra la ausencia de agua en la laguna Seca (11), debajo del tranque de Molles (7) y las Salinas (8), que permanecieron secas durante todo el invierno de 2007 (Figueroa et al., 2009). Esta situación compleja requiere estudios más precisos sobre el origen de los flujos que alimentan las lagunas de El Yali.

En el complejo El Yali parecen coexistir ecosistemas acuáticos cuyo origen del agua puede ser una combinación entre agua marina, agua subterránea y de escorrentía superficial y subsuperficial. Los dependientes del agua de escorrentía tienden a ser temporales y dulces, mientras que las cubetas más grandes y profundas tienden a ser salinas con fluctuaciones importantes de la salinidad en función de la importancia relativa del flujo de agua dominante (como aportes pluviométricos).

En relación al resto de variables físico-químicas medidas en el complejo El Yali, el pH mantiene correlaciones significativas con algunos iones pero negativas con todos los nutrientes. Aun- que el rango de variación del $\mathrm{pH}$ es estrecho $($ valor mínimo $=7.1$ y valor máximo $=9.3)$, los cuerpos de agua menos salinos también son los que presentan concentraciones más altas de nutrientes, lo cual puede estar relacionado con procesos de contaminación orgánica o descomposición de la materia orgánica.

En cuanto a los nutrientes, en los puntos de muestreo localizados en esteros o en sus desembocaduras, la fracción inorgánica del nitrógeno total es dominante respecto a la orgánica, al contrario de lo que ocurre en las lagunas y tranques muestreados, donde la fracción orgánica puede llegar a suponer más del $90 \%$ del total del nitrógeno disuelto (Fig. 4a). Esto concuerda con los resultados obtenidos por varios autores (e.g. Fellman et al., 2009), y se explica en relación con los usos del suelo (Pellerin et al., 2004). En nuestro caso, todas las lagunas con valores altos para la fracción orgánica del nitrógeno total están rodeadas de plantaciones de eucaliptos, los cuales en el proceso de descomposición de la hojarasca liberan materiales orgánicos y nutrientes ( $\mathrm{N}$ y $\mathrm{P}$ ) al agua (Corbeels et al., 2003; Goya et al., 2008). Los valores más altos de nitrógeno total se registraron en dos lagunas (13. Cabildo $=55.73 \mathrm{mg} / \mathrm{l}$ y 14 . El Rey $=14.55 \mathrm{mg} / \mathrm{l}$ ), en las que se han detectado floraciones extraordinarias de cianobacterias.

Dentro de la fracción inorgánica, las concentraciones de nitrato alcanzan valores muy elevados en varios humedales muestreados $(19.6 \mathrm{mg} / \mathrm{l}$ en Maitenlahue (16) y $14.86 \mathrm{mg} / \mathrm{l}$ en Mostazal (17)). Díaz-Valle et al. (2009) registraron tam- 
bién altas concentraciones de nitratos en 4 lagunas del complejo El Yali (Cabildo = $35.7 \mathrm{mg} / \mathrm{l}$, Matanzas $=9.92 \mathrm{mg} / \mathrm{l}, \mathrm{El}$ Rey $=30.43 \mathrm{mg} / \mathrm{l} \mathrm{y}$ Los Molles $=24.96 \mathrm{mg} / \mathrm{l}$ ) en otoño y verano de 2009. Las concentraciones de amonio más elevadas igualmente se detectaron en los mismos puntos de muestreo (5.99 mg/l en Maitenlahue (16) y $5.16 \mathrm{mg} / \mathrm{l}$ en Mostazal (17)). En cuanto al fósforo, los valores más elevados se registraron en la laguna Seca (11) (2.67 mg/l). Dado que esta laguna es temporal y es usada por el ganado para pastar, su inundación reciente supone procesos de redisolución tras la fase seca. Sin embargo, en más del $70 \%$ de los humedales muestreados se superan los $100 \mu \mathrm{g} / \mathrm{l}$ de SRP. Valores similares encuentran Díaz-Valle et al. (2009) en las cuatro lagunas de El Yali estudiadas (Cabil$\mathrm{do}=1.13 \mathrm{mg} / \mathrm{l}$, Matanzas $=0.13 \mathrm{mg} / \mathrm{l}, \mathrm{El}$ Rey $=$ $0.54 \mathrm{mg} / \mathrm{l}$ y Los Molles $=0.56 \mathrm{mg} / \mathrm{l})$. Estos valores se corresponden con sistemas eutróficos e hipertróficos (Vollenweider, 1968, Wetzel, 2001, Kalff, 2003) y, probablemente, es una consecuencia del incremento de las plantaciones de eucaliptos en las riberas y cuencas de drenaje de las lagunas del complejo, de las explotaciones ganaderas y de la entrada de vertidos orgánicas de la industria avícola.

La relación N/P suele ser utilizada para detectar la limitación de estos nutrientes en el medio acuático (e.g. Morris \& Lewis, 1988). En este caso, y dadas las altas concentraciones tanto de SRP como de nitratos registradas en todos los humedales estudiados, los valores por debajo del 16 para esta relación (Fig. 4d) estarían indicando la demanda de nitrógeno para equilibrar las altas concentraciones de fósforo. De hecho, en varias lagunas se observaron importantes afloramientos de cianobacterias (Díaz-Valle et al., 2009; Figueroa et al., 2009).

El análisis de las distintas variables físicoquímicas medidas en los humedales y cuerpos de agua del complejo El Yali permite hacer un diagnóstico sobre el estado actual de su gestión y conservación. Queda de manifiesto la complejidad hidrogeoquímica del complejo de humedales, pero se requieren estudios más precisos e intensivos para detectar los flujos que mantienen esta singularidad geoquímica. Sin embargo, el es- tado trófico de estos sistemas acuáticos se encuentra claramente alterado por el modelo de uso y gestión del complejo. Sería necesario diseñar y aplicar modelos de usos del territorio diferentes para el área de reserva y el resto que comprende el complejo de El Yali, definiendo los usos permitidos en cada caso. Pero esto puede resultar imposible si, como ocurre por ejemplo en la laguna de Matanzas, la mitad del cuerpo de agua queda dentro del límite propuesto para la reserva y el resto es de titularidad privada sin gestión alguna.

\section{AGRADECIMIENTOS}

Los resultados de este estudio han sido financiados por el proyecto A/010142/07 de la Agencia Española para la Cooperación Internacional (AECI). Se agradece al Centro Eula-Chile, de la Universidad de Concepción, por el apoyo logístico prestado, y a la corporación Nacional Forestal CONAF V, por permitirnos realizar investigación en los humedales bajo su protección.

\section{REFERENCIAS}

ALONSO, M. 1998. Las lagunas de la España peninsular. Limnetica, 15: 1-176.

APHA-AWWA-WPCF. 1985. Standard methods for the examination of water and wastewater. 16 ed. Washington. $769 \mathrm{pp}$.

BIBLIOREDES. 2009. Humedal el Yali. Dirección de Bibliotecas, Archivos y Museos. http://www. biblioredes.cl/BiblioRed/Nosotros+en+Internet/ Humedal+El+yali/hidro. (consulta: 10-10-2009).

BORGEL, R., 1983. Geografía de Chile. Tomo II. Geomorfología. Instituto Geográfico Militar. 182 pp.

BRITO, J. L. 1999. Listado conocido de los vertebrados del Humedal El Yali. http://www.biblioredes. $\mathrm{cl} /$ BiblioRed/Nosotros+en+Internet/Humedal+El +yali/reser (consulta: 10-10-2009).

CASADO, S. \& C. MONTES. 1995. Guía de los lagos y humedales de España. J.M. Reyero Editor. Madrid. $255 \mathrm{pp}$.

CLARKE, K. R. \& R. N. GORLEY. 2006. PRIMER v6: User Manual/Tutorial PRIMER-E. Plymouth.

COLE, A. C., R. P. BROOKS \& D. H. WARDROP. 1997. Wetland hydrology as a function of hidro- 
geomorphic (HGM) subclass. Wetlands, 17: 456467.

CONAF. 2009. Fauna silvestre humedal EL Yali. Corporación Nacional Forestal. http://www.conaf.cl/ ??page $=$ home $/$ contents \&seccion_id $=46312 \mathrm{fb} 63 \mathrm{bc}$ cd51f66219e 5d0fb20563\&unidad $=2$ \&pagina $=$. (Consulta: 10-10-2009).

CONAMA. 2005. Estrategia nacional para la conservación y uso racional de los humedales en Chile. Comisión Nacional de Medio Ambiente. Gobierno de Chile. 30 pp.

CORBEELS, M., A. M. O'CONNELL, T S. GROVE, D. S. MENDHAM \& S. J. RANCE. 2003. Nitrogen release from eucalypt leaves and legume residues as influenced by their biochemical quality and degree of contact with soil. Plant and Soil, 250: 15-28.

CUSTODIO, E. \& M. R. LLAMAS. 1983. Hidrogeología subterránea. Omega. Barcelona. 1157 pp.

DANIELS, A. E. \& G. S. CUMMING. 2008. Conversion or conservation? Understanding wetland change in Northwest Costa Rica. Ecol. Appl., 18: 49-63.

DGA. 2005. Evaluación de los recursos hídricos subterráneos Cuenca estero Yali bajo. Informe final.

DÍAZ-VALLE, K., C. GONZÁLEZ, S. SCOUT \& I. VILA. 2009. Humedales mediterráneos de la zona central de Chile: El Yali. VI Congreso Sociedad Chilena de Limnología. Coyhaique (Chile). 26-30 Octubre, 2009.

DINERSTEIN, E., D. M. OLSON, D. J. GRAHAM, A. L. WEBSTER, S. A. PRIMM, M. P. BOOKBINDER \& G. LEDEC. 1995. Una Evaluación del Estado de Conservación de las Ecorregiones de América Latina y el Caribe. Publ. Banco MundialFondo Mundial para la Naturaleza. Washington D.C. 135 pp.

DUSSAILLANT, A., P. GALDAMES C. SUN \&. 2009. Water level fluctuations in a coastal lagoon: El Yali Ramsar wetland, Chile. Desalination, 246 (1-3): 202-214.

FELLMAN, J. B., E. HOOD, D. V. D'AMORE, R. T. EDWARDS \& D. WHITE. 2009. Seasonal changes in the chemical quality and biodegradability of dissolved organic matter exported from soils to streams in coastal temperate rainforest watersheds. Biogeochemistry, 95: 277-293.

FERNÁNDEZ, J. V. 2004. Proposición de un plan de manejo integral de la Cuenca hidrográfica del estero Yali, V Región Metropolitana. Tesis para el título de Ingeniero Forestal. Escuela de Ingeniería
Forestal. Facultad de Ciencias Silvoagropecuarias. Universidad Mayor. Chile.

FIGUEROA, R., M. L. SUÁREZ, A. ANDREU, V. H. RUIZ \& M. R. VIDAL-ABARCA. 2009. Caracterización ecológica de humedales de la zona semiárida en Chile Central. Gayana, 73: 76-94.

GAJARDO, R. 1994. La Vegetación Natural de Chile. Clasificación y Distribución Geográfica. Editorial Universitaria, Santiago, Chile. 165 pp.

GONZÁLEZ-BERNÁLDEZ, F. G. \& J. M. REY BENAYAS, 1992. Geochemical relationships between groundwater and wetland soils and their effects on vegetation in central Spain. Geoderma, 55: 273-288.

GOYA, J. F., J. L. FRANGI, C. PÉREZ \& F. DALLA. 2008. Decomposition and nutrient release from leaf litter in Eucalyptus grandis plantations on three different soils in Entre Ríos, Argentina. Bosque, 29: 217-226.

GRIMM, N. \& S. G. FISHER. 1986. Nitrogen limitation potential of Arizona streams and rivers. $J$. Ariz.-Nev. Acad. Sci., 21: 31-43.

KALFF, J. 2003. Limnology. Inland Water Ecosystems. Prentice Hall. New Jersey. 592 pp.

KAZEZYILMAZ-ALHAN, C. M. \& M. A. MEDINA, Jr. 2008. The Effect of Surface/Ground Water Interactions on Wetland Sites with Different Characteristics. Desalination, 226: 298-305.

LEIVA, I., J. MEZA \& L. MÖDER. 1995. Fundamentos para la creación de la Reserva Nacional El Yali. CONAF V Región. Government Report. Boletín técnico $\mathrm{n}^{\circ} 59$.

LÓPEZ, P. 1984. Aguas salinas epicontinentales próximas a la costa mediterránea española. Estudio del medio. Tesis Doctoral. Universidad de Barcelona. $331 \mathrm{pp}$.

MACKERETH, F. J. K., J. HERON, J. TALLING. 1978. Water Analysis. Cumbria. Reino Unido. Freshwater Biological Association. Scientific publication $\mathrm{n}^{\mathrm{o}} 36.117 \mathrm{pp}$.

MARDONES, M. 1999. Contribución al conocimiento geomorfológico de las cuencas hidrográficas de los lagos Lanalhue y Lleu-Lleu. Rev. geogr. Chile, Terra Australis, 44: 87-106.

MARDONES, M. \& C. REUTHER. 1999. Geomorphological aspects of the drainage pattern around Lake Lanalhue and Lake Lleulleu in the active convergent margin setting of South-Central Chile. Mitt. Geol.-Palaeont. Inst. Univ. Hamburg, 83: 75-88. MARTINO, P. 1988. Limnología de las lagunas sali- 
nas españolas. Universidad Autónoma de Madrid. Tesis Doctoral. 264 pp.

MILLENIUM ECOSYSTEM ASSESSMENT. 2005.

Ecosystems and human well-being: wetlands and water synthesis. World Resources Institute. Washington, D.C. USA. 120 pp.

MITSCH, W. J. 2005. Applying science to conservation and restoration of the world's wetlands. Wat. Sci. Tech., 51: 13-26.

MITSCH, W. J. \& J. G. GOSSELINK. 2000. Wetlands. $3^{\text {rd }}$ Ed. John Wiley \& Sons. Inc., New York. $920 \mathrm{pp}$.

MORRIS, D. P. \& W. M. LEWIS. 1988. Phytoplankton nutrient limitation in Colorado mountain lakes. Freshwater Biology, 20: 315-327.

PARRA, O., C. VALDOVINOS, R. URRUTIA, M. CISTERNAS, E. HABIT \& M. MARDONES. 2003. Caracterización y tendencias tróficas de cinco lagos costeros de Chile Central. Limnetica, 22: 51-83.

PELLERIN, B. A., W. M. WOLHEIM, C. S. HOPKINSON, M. R. WILLIAMS, C. J. VOROSMARTY \& M. L. DALEY. 2004. Role of wetland and developed land use on dissolved organic nitrogen concentrations and DON/TDN in northeastern US rivers and streams. Limnol. Oceanogr., 49: 910-918.

PIPER, A. M. 1944. A graphic procedure in the geochemical interpretation of water analysis. Am. Geophys. Union Trans., 25: 914-923.

RUSSO, R. R. (Ed.). 2008. Wetlands. Ecology, conservation and restoration. Nova Science Publishers, Inc. New York. 446 pp.

SANTOS, I. R., M. I. MACHADO, L. F. NIENCHESKI, W. BURNETT, I. B. MILANI, C. F. F. AN-
DRADE, R. N. PETERSON, J. CHANTON \& P. BAISCH. 2008. Major ion chemistry in a freshwater coastal lagoon from southern Brazil (Mangueira Lagoon): Influence of groundwater inputs. Aquat. Geochem., 14: 133-146.

TOTH, J. 1984. The role of regional gravity flow in the chemical and termal evolution of groundwater. Proc. $1^{e r}$ Canadian-American Conference on Hydrogeology.

VICTORIANO, P. F., A. L. GONZÁLEZ \& R. SCHLATTER. 2006. Estado del conocimiento de las aves de aguas continentales de Chile. Gayana, 70: 140-160.

VILINA, Y. \& M. LÓPEZ. 1996. The neotropical plovers of Estero El Yali in Central Chile. In: Shorebird Ecology and Conservation in the Western Hemisphere. P. Hicklin (ed.): 85-92. International Waters Studies 8.

VOLLENWEIDER, R. A. 1968. Scientific fundamentals of the eutrophication of lakes and flowing waters, with particular reference to nitrogen and phosphorus as factors in eutrophication. OECD Report n. DAS/CSI/68.27. Paris. OECD.

WETZEL, R. G. 2001. Limnology. Lakes and river ecosystems. $3^{\text {rd }}$ Ed. Academic Press. San Diego. $1006 \mathrm{pp}$.

ZEDLER, J. B. \& S. KERCHER. 2005. Wetland resources: status, trends, ecosystem service and restorability. Annu. Rev. Environ. Resour., 30: 39-74.

ZEDLER, J. B. \& J. M. WEST. 2008. Declining diversity in natural and restored salt marshes: A 30-year study of Tijuana Estuary. Restoration Ecology, 16: 249-262. 